\title{
MÉTODOS DE INDEXAÇÃO DE INDICADORES NA AVALIAÇÃO DA QUALIDADE DO SOLO EM RELAÇÃO À EROSÃO HÍDRICA
}

\author{
Bernardo Moreira Cândido(1), Marx Leandro Naves Silva(2)*, Nilton Curi(2), Diego Antônio \\ França de Freitas( ${ }^{(3)}$, Ronaldo Luiz Mincato(4) e Mozart Martins Ferreira(2)
}

(1) Universidade Federal de Lavras, Departamento de Ciência do Solo, Programa de Pós-Graduação em Ciência do Solo, Lavras, Minas Gerais, Brasil.

(2) Universidade Federal de Lavras, Departamento de Ciência do Solo, Lavras, Minas Gerais, Brasil.

(3) Universidade Federal de Viçosa, Instituto de Ciências Agrárias, Campus de Florestal, Florestal, Minas Gerais, Brasil.

(4) Universidade Federal de Alfenas, Instituto de Ciências da Natureza, Alfenas, Minas Gerais Brasil.

* Autor correspondente.

E-mail: marx@ufla.br

\section{RESUMO}

A avaliação da qualidade dos solos agrícolas é importante para definição e adoção de práticas de manejo que garantam a sustentabilidade socioeconômica e ambiental. Os métodos de indexação dos indicadores de qualidade denominados Índice de Qualidade Integrado (IQI) e Índice de Qualidade Nemoro (IQN) foram utilizados neste estudo para avaliar a qualidade de solo em áreas experimentais de plantio de eucalipto. A seleção dos indicadores foi feita a partir de nove indicadores de qualidade do solo: diâmetro médio geométrico, permeabilidade à água, matéria orgânica, macro e microporosidade, volume total de poros, densidade do solo, resistência à penetração e índice de floculação, que estão relacionados à erosão hídrica. Os tratamentos constituíram de eucalipto plantado em nível, com e sem a manutenção dos resíduos, em desnível e solo descoberto, em dois biomas distintos, cujas vegetações nativas são Cerrado e Floresta. Os índices de qualidade do solo (IQS) apresentaram alta correlação com a erosão hídrica. Entre os sistemas manejados, o Eucalipto com manutenção do resíduo evidenciou valores mais elevados em ambos os índices, ressaltando-se a importância da cobertura vegetal e manutenção da matéria orgânica para conservação do solo e da água em sistemas florestais. Os IQS demonstraram alto coeficiente de correlação inversa com as perdas de solo e água. Em locais com as maiores taxas de erosão hídrica manifestaram também os menores valores de IQI e IQN. Assim, os índices testados permitiram avaliar com eficácia os efeitos dos manejos adotados sobre a qualidade do solo em relação à erosão hídrica.

Palavras-chave: índice de qualidade integrado, índice de qualidade Nemoro, sustentabilidade, eucalipto, conservação ambiental. 


\title{
ABSTRACT: INDICATOR INDEXING METHODS IN ASSESSMENT OF SOIL QUALITY IN RELATION TO WATER EROSION
}

\begin{abstract}
Assessing the quality of agricultural soils is important for defining and adopting management practices that ensure socioeconomic and environmental sustainability. The methods for indexation of quality indicators called the Integrated Quality Index (IQI) and the Nemoro Quality Index (NQI) were used in this study to evaluate soil quality in experimental plots planted to eucalyptus. The selection of these indicators was made based on nine soil quality indicators: geometric mean diameter, water permeability, organic matter, macro- and microporosity, total porosity, bulk density, penetration resistance, and flocculation index, which are related to water erosion. Treatments consisted of eucalyptus planted on level land, with and without maintenance of residues on the soil surface, planted on a downslope, and planted on bare soil in two distinct biomes, whose native vegetation are Cerrado (Brazilian tropical savanna) and Forest. The soil quality indices (SQI) were highly correlated with erosion. Among the management systems, Eucalyptus with maintenance of the residues had higher values in both indices, highlighting the importance of plant cover and organic matter for soil and water conservation in forest systems. The SQI had a high inverse correlation coefficient with soil and water losses. Places with the highest rates of water erosion also had the lowest IQI and NQI values. Thus, the indices tested allowed efficient evaluation of the effects of the management practices adopted on soil quality in relation to water erosion.
\end{abstract}

Keywords: integrated quality index, Nemoro quality index, sustainability, eucalyptus, environmental conservation.

\section{INTRODUÇÃO}

A partir da década de 1990, o conceito de qualidade do solo tem recebido atenção, pois, da mesma forma que o ar e a água, ela influencia no equilíbrio e na produtividade de determinado ecossistema e dos ambientes adjacentes. Contudo, ao contrário do ar e da água que possuem padrões de qualidade, a qualidade do solo tem sido difícil de definir e quantificar. De acordo com Doran e Parkin (1994), a qualidade do solo é característica abstrata e não pode ser definida, pois depende de fatores externos como uso da terra, práticas de manejo, interações entre o ambiente e o ecossistema, prioridades políticas e socioeconômicas e assim por diante. A concepção do que constitui bom solo depende do objetivo do trabalho. Entretanto, para manejar e manter os solos em bom estado para as futuras gerações, a qualidade desses deve ser definida de forma a abranger as facetas das suas funções.

A qualidade do solo tem sido definida de várias maneiras. Entretanto, a definição mais completa da qualidade do solo é descrita por Karlen et al. (1998), como sendo a capacidade de o solo funcionar para sustentar a produtividade animal e vegetal, manter ou melhorar a qualidade da água e do ar e prover moradia e saúde humana.

A dificuldade em avaliar o solo é um dos principais obstáculos para o manejo sustentável dos seus ecossistemas (Nuria et al., 2011). Diante disso, diversos métodos de avaliação da qualidade do solo têm sido desenvolvidos, como projetos de cartão de solo e kits de teste (Ditzler e Tugel, 2002), métodos geoestatísticos (Sun et al., 2003) e índices de qualidade do solo (IQS) (Armenise et al., 2013). Entre esses, os IQS são os mais comumente utilizados, pela facilidade de uso, por serem quantitativamente flexíveis, além de poderem ser utilizados como suporte para avaliação do manejo do solo e dos ecossistemas.

Dessa forma, os IQS têm sido importantes para avaliação dos impactos das práticas agrícolas em sistemas florestais e pastagens (Freitas et al., 2012), produção de culturas (Andrews et al., 2002), manejo de resíduos orgânicos (Andrews e Carroll, 2001) e do solo em escala regional (Brejda et al., 2000a,b).

No entanto, grande número de atributos do solo precisa ser determinado para avaliar a qualidade do solo. Indicadores físicos e químicos têm sido utilizados, pois as análises são simples e os métodos disponíveis (Bhardwaj et al., 2011). Dependendo da finalidade para a qual os IQS estão sendo obtidos, extensa lista de atributos do solo pode ser necessária. Por isso, para determinadas finalidades, é preciso selecionar indicadores que interferem na função que está sendo avaliada e que sejam mensuráveis e sensíveis para detectar as diferenças espaciais e, ou, temporais (Karlen et al., 1997).

O cálculo dos IQS parte da definição de indicadores de qualidade do solo, ou seja, dos processos e das propriedades do solo sensíveis a mudanças causadas por fatores naturais e antrópicos (Doran e Jones, 1996). Assim, os indicadores de qualidade são propriedades físicas, químicas e biológicas do solo capazes de serem alteradas rapidamente em resposta às variações nas condições do solo (Brejda et al., 2000a; Marzaioli et al., 2010). Entretanto, os indicadores podem ser combinados de diversas formas com índices aditivos (Andrews e Carroll, 2001), por pesos (Karlen et al., 1998), ou multiplicativos (Doran e Parkin, 1994). O Índice de Qualidade Integrado (IQI) e o Índice de Qualidade Nemoro (IQN) são exemplos desses cálculos. 
O IQI, desenvolvido a partir dos IQS de Doran e Parkin (1994), combina os valores de peso dos indicadores selecionados em um índice, utilizando equação com sistema de pontuação simples. Diferentemente, o IQN, desenvolvido por Nemoro (Qin e Zhao, 2000), é com base na média e no valor mínimo do indicador, sem considerar seu peso. Nesse caso, os resultados são influenciados pelo mínimo do indicador, de forma análoga à lei do mínimo na produção agrícola (van der Ploeg et al., 1999). A disparidade entre as metodologias utilizadas para indexação dos indicadores de qualidade do solo e modelos leva a questões sobre se a aplicação de vários índices produziria resultados diferentes. No entanto, oportunidades para a comparação entre os índices são raras, pois normalmente não se dispõem mais de uns IQS para uma área em particular.

Os sistemas florestais, especialmente as florestas plantadas de eucalipto, estão situados em ecossistemas sensíveis às perturbações antrópicas em razão das plantações em relevo acidentado, dos solos com baixa fertilidade natural e da grande parte das plantações estabelecidas em antigas áreas agrícolas e de pastagens degradadas. Assim, a quantificação da qualidade do solo pode constituir em instrumento para monitorar a sustentabilidade do sistema de produção, pois permite caracterizar a situação atual, alertar para situações de possíveis riscos e prever situações futuras; ou seja, identificar problemas no sistema de manejo, demonstrando se contribui para alterar a capacidade produtiva do solo, bem como sugerir a necessidade de adoção de práticas conservacionistas para mitigação dos impactos deletérios decorrentes do sistema de plantio.

Dessa forma, o objetivo deste estudo foi gerar IQS por dois métodos de indexação dos indicadores de qualidade, IQI e IQN, para avaliar a acurácia deles na discriminação dos diferentes tratamentos em relação à erosão hídrica.

\section{MATERIAL E MÉTODOS}

O trabalho foi realizado em solos sob plantios de eucalipto em duas sub-bacias hidrográficas, denominadas hortos florestais Matão e Barra do Moeda, na bacia hidrográfica do rio Paraná, município de Três Lagoas, MS (20 $45^{\circ} \mathrm{S}$ e $\left.51^{\circ} 40^{\prime} \mathrm{W}\right)$ (Figura 1). O clima na região é o tropical quente e úmido (Aw), conforme Köppen (Sparovek et al., 2007). Nas duas áreas, os solos dominantes foram classificados, respectivamente, como Latossolo Vermelho distrófico típico, textura média-alta, fase floresta (LVd1) e Latossolo Vermelho distrófico típico, textura média-baixa, fase cerrado (LVd2).

Os sistemas adotados no LVd1 foram solo descoberto (SD), plantio de eucalipto em nível sem (ES) e com (EC) resíduo e floresta nativa (FN).
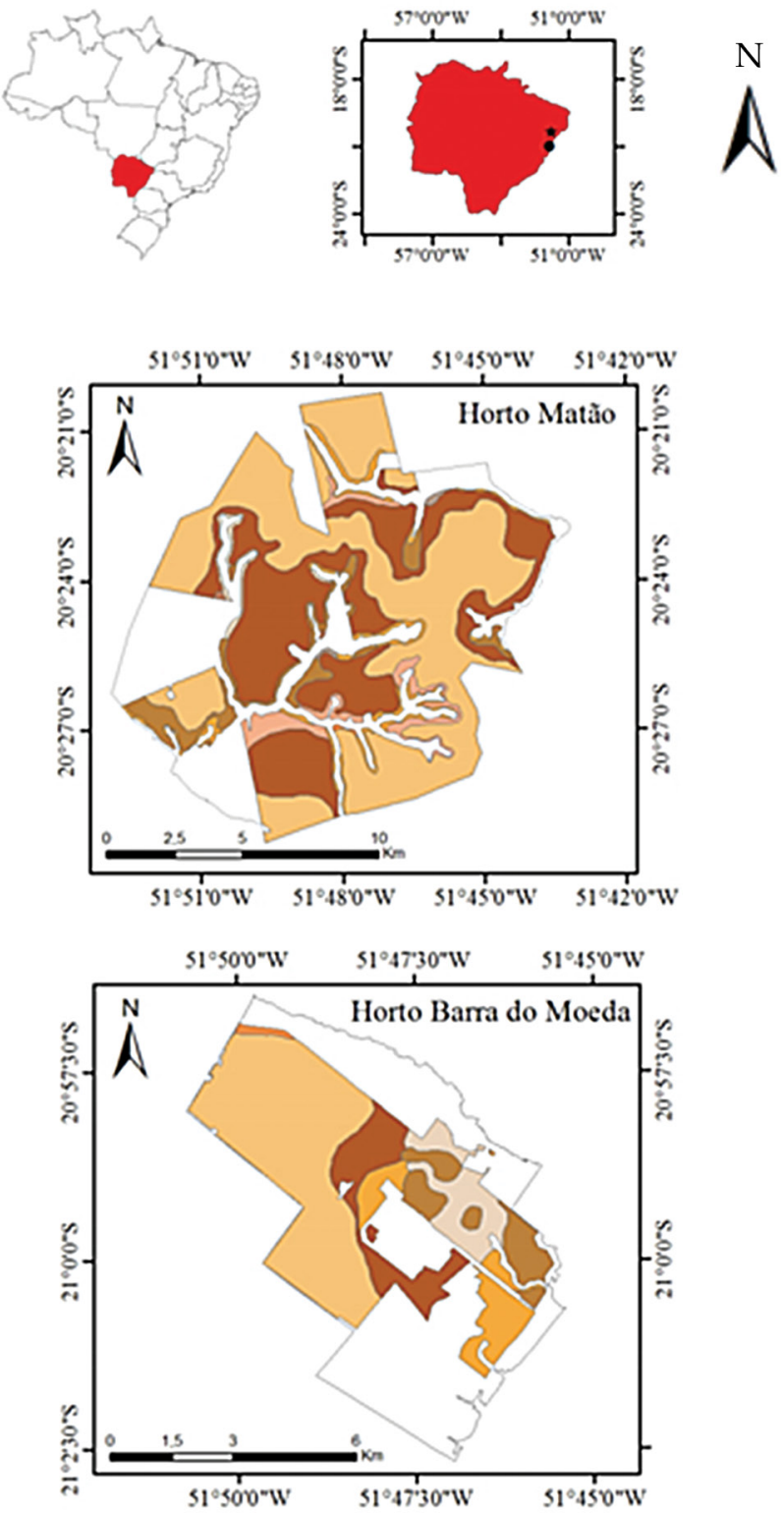

Legenda

* Horto Matão

- Horto Barra do Moeda

Mato Grosso do Sul

Classes de solo

$\square$ LVd1

LVd2

Figura 1. Sub-bacias estudadas e abrangência do Latossolo Vermelho distrófico típico, textura média-alta, fase floresta (LVd1) e Latossolo Vermelho distrófico típico, textura média-baixa, fase cerrado (LVd2).

No LVd2, os sistemas foram solo descoberto (SD), plantio de eucalipto em nível sem (ES) e com (EC) resíduo, plantio em desnível (ED) e 
cerrado nativo $(\mathrm{CN})$. Os tratamentos EC foram conduzidos no sistema de cultivo mínimo, com pouca movimentação do solo e manutenção de resíduo na superfície.

Os valores dos atributos físicos e matéria orgânica do solo (MOS), utilizados como indicadores da qualidade do solo, são descritos no quadro 1. Os indicadores foram selecionados com base no CMD, considerando o objetivo de manejo a conservação do solo e da água.

Em razão da diferença de unidades entre os indicadores, foram utilizadas funções de pontuação padrão (FPP, Quadro 2), com cada indicador variando entre 0 e 1 . A partir da sensibilidade do indicador da qualidade do solo, foram aplicadas funções do tipo: "mais é melhor e menos é melhor", se a melhor funcionalidade do solo foi associada a valores altos ou baixos, respectivamente (Liebig et al., 2001).

Os valores máximos e mínimos de cada indicador foram considerados como limites superiores e inferiores (Alvarenga et al., 2012; Rahmanipour et al., 2014). Os valores assim obtidos foram usados nas FPP (Quadro 2) para normalizar [N(x)] os resultados de cada indicador em cada amostra de solo.

Para obter o IQI, foram atribuídos pesos aos indicadores de acordo com a relevância de cada um para a conservação do solo e da água. À matéria orgânica do solo (MOS), foi atribuído peso 0,2 ,

Quadro 1. Atributos físicos e matéria orgânica de duas classes de solo sob diferentes sistemas de manejo no município de Três Lagoas, MS

\begin{tabular}{|c|c|c|c|c|c|c|c|c|c|}
\hline \multirow{2}{*}{ Atributo } & \multicolumn{4}{|c|}{ LVd1 } & \multicolumn{5}{|c|}{ LVd2 } \\
\hline & FN & EC & ES & SD & $\mathrm{CN}$ & EC & ED & ES & SD \\
\hline Argila $\left(\mathrm{g} \mathrm{kg}^{-1}\right)$ & 250 & 280 & 300 & 310 & 160 & 230 & 210 & 220 & 220 \\
\hline Silte $\left(\mathrm{g} \mathrm{kg}^{-1}\right)$ & 40 & 60 & 60 & 70 & 50 & 40 & 30 & 30 & 40 \\
\hline Areia total $\left(\mathrm{g} \mathrm{kg}^{-1}\right)$ & 710 & 660 & 640 & 620 & 790 & 730 & 760 & 750 & 740 \\
\hline Densidade do solo $\left(\mathrm{kg} \mathrm{dm}^{-3}\right)$ & 1,36 & 1,32 & 1,37 & 1,44 & 1,47 & 1,48 & 1,51 & 1,51 & 1,57 \\
\hline Volume total de poros $\left(\mathrm{m}^{3} \mathrm{~m}^{-3}\right)$ & 0,49 & 0,51 & 0,46 & 0,46 & 0,45 & 0,44 & 0,43 & 0,42 & 0,4 \\
\hline Macroporosidade $\left(\mathrm{m}^{3} \mathrm{~m}^{-3}\right)$ & 0,3 & 0,2 & 0,15 & 0,15 & 0,27 & 0,19 & 0,18 & 0,19 & 0,19 \\
\hline Microporosidade $\left(\mathrm{m}^{3} \mathrm{~m}^{-3}\right)$ & 0,19 & 0,31 & 0,31 & 0,31 & 0,18 & 0,25 & 0,26 & 0,23 & 0,21 \\
\hline Índice de floculação (\%) & 64 & 59 & 48 & 51 & 80 & 73 & 80 & 69 & 60 \\
\hline Diâmetro médio geométrico $(\mathrm{mm})$ & 4,78 & 3,13 & 3,1 & 1,51 & 3,56 & 3,48 & 4,07 & 3,61 & 3,42 \\
\hline Permeabilidade $\left(\mathrm{mm} \mathrm{h}^{-1}\right)$ & $3.829,8$ & 865,5 & 435,3 & 190,4 & $1.329,3$ & 256,3 & 512,3 & 282 & 46 \\
\hline Matéria orgânica do solo (g kg-1) & 63,6 & 66,4 & 55,0 & 44,5 & 30,9 & 45,0 & 41,5 & 40,0 & 29,5 \\
\hline Resistência do solo à penetração $(\mathrm{MPa})$ & 2,6 & 3,3 & 3,5 & 3,8 & 2,8 & 3,0 & 3,1 & 3,3 & 3,4 \\
\hline
\end{tabular}

Latossolo Vermelho distrófico típico textura média-alta fase floresta (LVd1), Latossolo Vermelho distrófico típico textura média-baixa fase cerrado (LVd2). SD: solo descoberto; ES: plantio em nível sem resíduo; EC: plantio em nível com resíduo; ED: plantio em desnível (ED); CN: cerrado nativo; e FN: floresta nativa.

Quadro 2. Funções de pontuação padrão e parâmetros indicadores em duas classes de solo no município de Três Lagoas, MS

\begin{tabular}{|c|c|c|c|c|c|c|c|c|}
\hline \multirow{2}{*}{ Indicador } & \multirow{2}{*}{ Tipo de função(1) } & \multicolumn{2}{|c|}{ LVd1 } & \multicolumn{2}{|c|}{ LVd2 } & \multicolumn{2}{|c|}{ FPP(2) } & \multirow{7}{*}{$\begin{array}{l}x \leq L \\
L<x<U \\
\mathrm{x} \geq \mathrm{U}\end{array}$} \\
\hline & & $\mathbf{L}$ & $\mathbf{U}$ & $\mathbf{L}$ & $\mathbf{U}$ & & & \\
\hline DMG & MB & 1,25 & 4,8 & 2,21 & 4,61 & \multirow{5}{*}{\multicolumn{2}{|c|}{$\mathrm{N}(x)=\left\{\begin{array}{c}0,1 \\
0,9 \frac{x-L}{U-L}+0,1 \\
1\end{array}\right.$}} & \\
\hline Ks & $\mathrm{MB}$ & 184 & 3860 & 41,00 & $1.336,00$ & & & \\
\hline MOS & MB & 18,70 & 39,90 & 16,40 & 28,70 & & & \\
\hline IF & MB & 44,83 & 66,67 & 56,52 & 90,48 & & & \\
\hline VTP & MB & 44,1 & 52,8 & 37,60 & 47,60 & & & \\
\hline Macro & $\mathrm{MB}$ & 10,1 & 33,4 & 11,80 & 28,30 & 1 & & $x \leq L$ \\
\hline Ds & $\mathrm{LB}$ & 1,25 & 1,48 & 1,39 & 1,66 & & $x-L$ & $L<x<U$ \\
\hline $\mathrm{RP}$ & $\mathrm{LB}$ & 1,70 & 5,35 & 2,06 & 3,24 & 0,9 & $\overline{U \cdot L}$ & $\mathrm{x} \geq \mathrm{U}$ \\
\hline Micro & $\mathrm{LB}$ & 17,40 & 34,00 & 15,80 & 29,40 & 0,1 & & \\
\hline
\end{tabular}

(1) Tipos de funções: mais é melhor (MB) e menos é melhor (LB). (2) Funções de Pontuação Padrão (FPP), nessas duas equações x é o valor do indicador; $\mathrm{N}(\mathrm{x})$ é a pontuação do indicador variando entre 0,1 e 1 ; e L e U são os valores dos limites inferiores e superiores, respectivamente. Latossolo Vermelho distrófico típico textura média-alta fase floresta (LVd1); e Latossolo Vermelho distrófico típico textura média-baixa fase cerrado (LVd2). 
o dobro em relação aos demais indicadores, por causa da elevada importância nos processos de retenção e armazenamento de água e de crescimento vegetal. Ao restante dos indicadores, foi atribuído, igualmente, peso 0,1 .

O IQI e o IQN foram calculados para todos os tratamentos nas duas classes de solos, LVd1 e LVd2, de acordo com as seguintes equações:

$$
I Q I=\sum_{i=1}^{n} W_{i} N_{i}
$$

em que $\mathrm{W}_{\mathrm{i}}$ é o peso de cada indicador; e $\mathrm{N}_{\mathrm{i}}$, a pontuação do indicador.

$$
I Q I=\sqrt{\frac{P_{m e d}^{2}+P_{\min }^{2}}{2}} \times \frac{n-1}{n}
$$

em que $\mathrm{P}_{\text {med }}$ é a média; $\mathrm{P}_{\text {mín }}$ o mínimo das pontuações dos indicadores selecionados em cada amostra de cada tratamento; e $n$, é o número de indicadores.

Para relacionar os IQS com a erosão hídrica, foram utilizados dados de perdas de solo e água. As parcelas para avaliar as perdas de solo e água foram instaladas no campo, com dimensões de $4,0 \times 24 \mathrm{~m}$ para os tratamentos com solo descoberto, vegetação nativa e eucalipto sem resíduo/desnível; e $14 \times 24 \mathrm{~m}$, nos tratamentos sob eucalipto com resíduo. As parcelas foram contornadas com chapas galvanizadas de $40 \mathrm{~cm}$ de altura, enterradas à profundidade de $20 \mathrm{~cm}$. O comprimento da parcela acompanha o sentido do declive do terreno. À jusante das parcelas, foram colocadas calhas coletoras, das quais saem canos de PVC de 3" para condução das enxurradas aos tanques coletores. O sistema coletor consistiu de tanque de sedimentação de $500 \mathrm{~L}$ e tanque coletor de água e sedimentos de $250 \mathrm{~L}$. Entre os dois tanques havia um divisor do tipo Geib com
15 janelas para que, após o enchimento do tanque de sedimentação, apenas 1/15 da enxurrada passasse para o tanque coletor.

Foi realizado teste de médias entre os índices dentro dos tratamentos em cada região, pelo teste Scott-Knott. Visando estabelecer uma correlação entre os atributos do solo, as perdas de solo e água e os índices de qualidade, em cada tratamento, prosseguiram-se com a análise de componentes principais (ACP). O IQI e o IQN foram considerados variáveis adicionais às perdas de solo e água, atributos físicos e MOS.

Além disso, para obter um coeficiente de correlação, foram feitas relações lineares entre a média dos índices e os valores de perdas de solo e água. Essa técnica foi utilizada também para verificar o grau de correlação entre IQI e IQN.

\section{RESULTADOS E DISCUSSÃO}

Os dois métodos de indexação dos indicadores (IQI e IQN) foram eficientes em reproduzir a variação da qualidade do solo nos dois sistemas de uso e manejo. Qi et al. (2009) e Rahmanipour et al. (2014), aferindo a eficácia desses dois índices, obtiveram melhor estimativa da qualidade do solo com o IQI ao invés do IQN, que poderia ser explicada pelo peso do indicador como fator de discriminação no IQI em contraste ao IQN. Entretanto, neste estudo, não foi observada diferença significativa entre os índices. A relação linear entre os índices comprovou alta correlação, com $\mathrm{R}^{2}=0,942$ (Figura 2). Dessa forma, optou-se pela utilização das médias de IQI e IQN para conferir a correlação existente entre os IQS e a erosão hídrica (Figura 3).

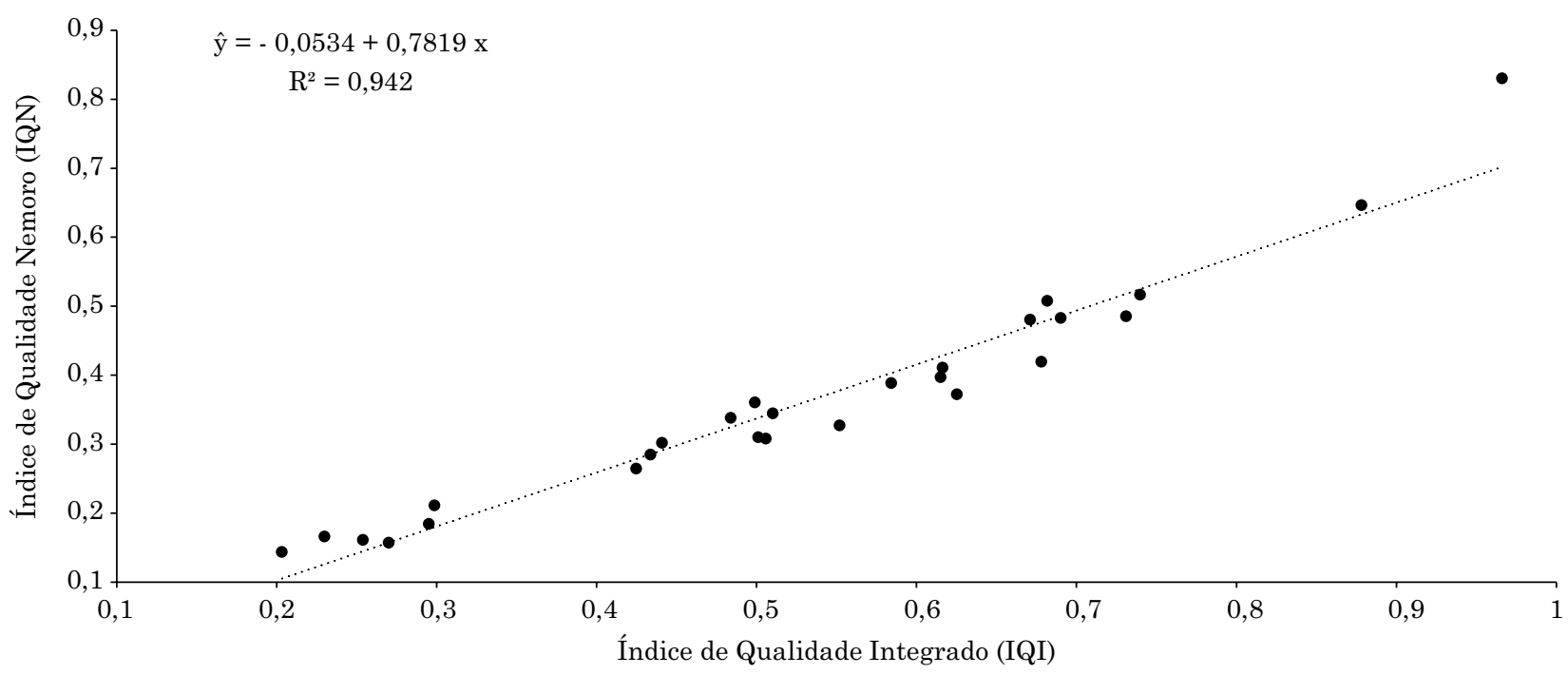

Figura 2. Relação linear entre os índices de qualidade do solo Nemoro (IQN) e integrado (IQI). 

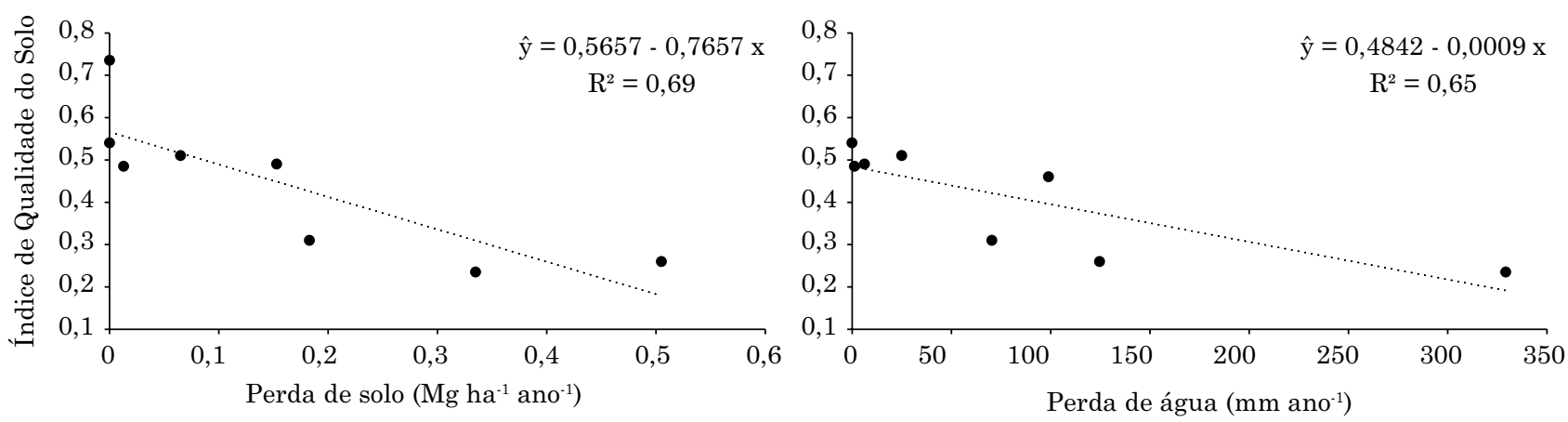

Figura 3. Relações lineares entre a erosão hídrica (perdas de solo e de água) e o índice de qualidade do solo.

As perdas de solo e água se comportaram de forma inversa à qualidade do solo nos tratamentos estudados. Essa relação é confirmada pelos elevados coeficientes lineares obtidos entre a erosão hídrica e os IQS (Figura 3), que demonstram a importância do estudo de avaliação da qualidade do solo para definir práticas de manejo conservacionistas nos sistemas florestais.

Os resultados dos índices de qualidade no LVd1 foram superiores no ambiente nativo (FN), no que diz respeito à qualidade do solo, em relação aos demais tratamentos, com IQI de 0,86 e IQN de 0,66 . Os índices distinguiram três grupos de acordo com a qualidade do solo, em ordem decrescente: FN $>$ EC $>$ ES, SD (Quadro 3). Isso indica a degradação do ambiente a partir do momento em que sai do equilíbrio natural e começa a ser manejado. Resultados semelhantes foram obtidos por Freitas et al. (2012), os quais avaliaram a qualidade do solo sob sistema florestal em Latossolos. Alvarenga et al. (2012) observaram maiores valores de IQS, para recarga de água em áreas de Mata Atlântica, ratificando que os fatores uso e manejo do solo são determinantes para a infiltração de água no solo.

Entre os tratamentos com eucalipto no LVd1, os resultados do IQI e IQN evidenciaram a influência positiva da cobertura vegetal na conservação do solo e da água, em que o EC apresentou índice apenas inferior ao da vegetação nativa ( $\mathrm{FN})$ (Quadro 3). Isso demonstra que o cultivo mínimo aumenta os IQS e que esse sistema deveria ser adotado em detrimento aos plantios convencionais.

De acordo com Rahmanipour et al. (2014), os índices obtidos para EC podem ser classificados como alto e médio, para o LVd1 e LVd2, respectivamente, em contraste aos baixos valores obtidos no tratamento ES, em ambas as classes de solo. Isso ocorre, pois a manutenção do resíduo aumenta a estabilidade de agregados, a retenção e infiltração de água no solo e a porosidade (Conforti et al., 2013; Mabit e Bernard, 2010), atributos que são indicadores da qualidade do solo e contribuem para a elevação dos índices.

Quadro 3. Índices de qualidade integrado (IQI), Nemoro (IQN), do solo médio e valores de perda de solo e água para duas classes de solos no município de Três Lagoas, MS

\begin{tabular}{|c|c|c|c|c|c|}
\hline Tratamento & IQI & IQN & IQS $_{\text {médio }}$ & Perda de Solo & Perda de Água \\
\hline & & & & $\mathrm{Mg} \mathrm{ha}^{-1} \mathrm{ano}^{-1}$ & $\mathrm{~mm}$ ano-1 \\
\hline & \multicolumn{5}{|c|}{ LVd1 } \\
\hline $\mathrm{SD}$ & $0,27 \mathrm{c}$ & $0,17 \mathrm{c}$ & 0,22 & 0,51 & 124,7 \\
\hline ES & $0,39 \mathrm{c}$ & $0,24 \mathrm{c}$ & 0,32 & 0,18 & 70,4 \\
\hline $\mathrm{EC}$ & $0,63 \mathrm{~b}$ & $0,40 \mathrm{~b}$ & 0,51 & 0,01 & 1,2 \\
\hline \multirow[t]{2}{*}{ FN } & $0,86 \mathrm{a}$ & $0,66 \mathrm{a}$ & 0,76 & 0,00 & 3,1 \\
\hline & \multicolumn{5}{|c|}{$\operatorname{LVd} 2$} \\
\hline $\mathrm{SD}$ & $0,31 \mathrm{c}$ & $0,22 \mathrm{~b}$ & 0,27 & 0,34 & 329,3 \\
\hline ES & $0,47 \mathrm{~b}$ & $0,31 \mathrm{~b}$ & 0,39 & 0,85 & 98,9 \\
\hline ED & $0,58 \mathrm{a}$ & $0,40 \mathrm{a}$ & 0,48 & 0,15 & 6,2 \\
\hline $\mathrm{EC}$ & $0,59 \mathrm{a}$ & $0,38 \mathrm{a}$ & 0,49 & 0,07 & 25,0 \\
\hline $\mathrm{CN}$ & $0,70 \mathrm{a}$ & $0,49 \mathrm{a}$ & 0,60 & 0,00 & 0,0 \\
\hline
\end{tabular}

Latossolo Vermelho distrófico típico textura média-alta fase floresta (LVd1); Latossolo Vermelho distrófico típico textura média-baixa fase cerrado (LVd2). SD: solo descoberto; ES: plantio em nível sem resíduo; EC: plantio em nível com resíduo; ED: plantio em desnível (ED); CN: cerrado nativo; FN: floresta nativa. IQI: índice de qualidade integrado. IQN: índice de qualidade Nemoro. Valores seguidos pela mesma letra nas colunas, dentro de cada região, não diferem estatisticamente entre si pelo teste Scott-Knott, a $5 \%$. 
Em LVd2, onde a vegetação nativa é o Cerrado, o IQI e o IQN demonstraram a superioridade dos tratamentos CN, ED e EC em relação a ES e SD. Isso destaca que o manejo do eucalipto com manutenção do resíduo, independentemente se é em nível ou desnível, proporciona elevados IQS, igualando-se ao ambiente de referência, no caso CN. Valores próximos foram obtidos por Freitas et al. (2012), os quais, trabalhando com Latossolo Vermelho em ambiente de Cerrado, obtiveram valores de IQS em torno de 0,61, 0,65 e 0,58 para $\mathrm{CN}, \mathrm{ED}$ e EC, respectivamente.

Os resultados apresentados pela ACP (Figuras 4 e 5) demonstraram elevada correlação entre os índices avaliados e as perdas de solo e água (Quadro 3). Isso pode ser confirmado pelos altos valores de $\mathrm{R}^{2}$ obtidos nas relações lineares entre a média dos índices IQI e IQN e as perdas de solo e água, resultando em correlação inversa (Figura 3). Essa forte relação entre os IQS e a erosão hídrica ocorre, pois a erosão interfere negativamente os

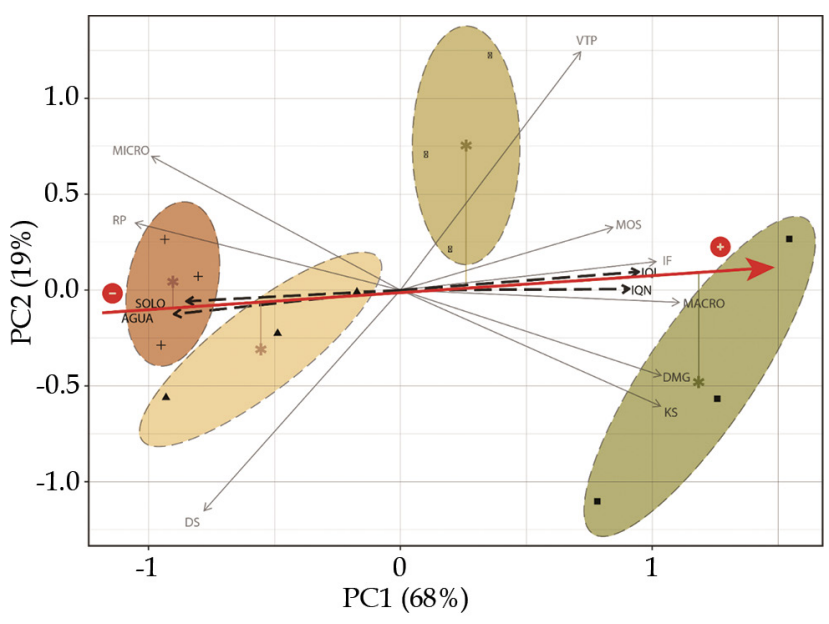

Tratamento

$\& \mathrm{EC} \Delta \mathrm{ES} \boldsymbol{\mathrm { FN }}+\mathrm{SD}-$ Qualidade do solo

Figura 4. Análise dos componentes principais para os atributos físicos e matéria orgânica do solo (MOS) na classe de solo LVd1. Plantio em nível com resíduo (EC); plantio em nível sem resíduo (ES); floresta nativa $(\mathrm{FN})$; solo descoberto (SD); microporosidade (MICRO); macroporosidade do solo (MACRO); resistência do solo à penetração (RP); densidade do solo (DS); volume total de poros (VTP); índice de floculação (IF); diâmetro médio geométrico de agregados do solo (DMG); permeabilidade do solo a água (KS); perda de solo (SOLO); perda de água (ÁGUA); índice de qualidade integrado (IQI); índice de qualidade Nemoro (IQN); componente principal 1 (PC1); e componente principal 2 (PC2). atributos do solo utilizados como indicadores de qualidade, como o teor de MOS (Rhoton e Tyler, 1990), a densidade do solo (Frye et al., 1982) e a capacidade de retenção de água (Nizeyimana e Olson, 1988), entre outros. Dessa forma, as componentes principais evidenciaram com eficiência o gradiente de qualidade do solo e da erosão hídrica entre os sistemas de manejo de eucalipto avaliados, a vegetação nativa e o solo descoberto (Figuras 4 e 5).

Ao projetar cada grupo na reta de qualidade do solo (Figuras 4 e 5), obteve-se que o ambiente nativo é o que apresenta maior qualidade do solo, seguido pelo eucalipto com a manutenção dos resíduos. Isso comprova o importante papel desempenhado pela MOS na sustentabilidade dos sistemas agrícolas, influenciando os atributos físicos, químicos e biológicos do solo, com reflexos na estabilidade da produtividade dos sistemas de produção. Isso corrobora o fato de que sistemas florestais bem manejados tendem a apresentar comportamento semelhante à vegetação nativa, no que diz respeito

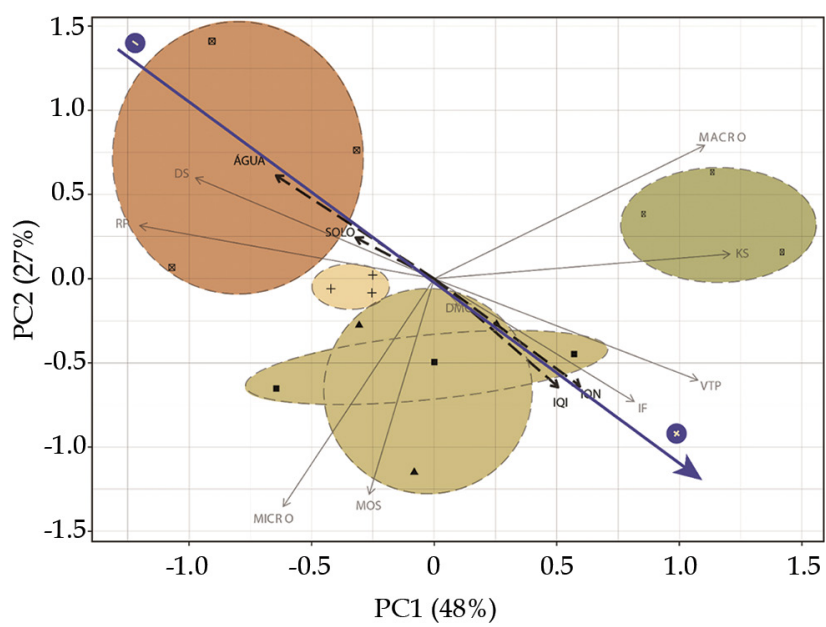

Tratamento

\& $\mathrm{CN} \Delta \mathrm{EC}$ - ED + ES - Qualidade do solo

Figura 5. Análise dos componentes principais para os atributos físicos e matéria orgânica do solo (MOS) na classe de solo LVd2. Plantio em nível com resíduo (EC); plantio em nível sem resíduo (ES); plantio em desnível com resíduo (ED); Cerrado nativo (CN); solo descoberto (SD); microporosidade (MICRO); macroporosidade do solo (MACRO); resistência do solo à penetração (RP); densidade do solo (DS); volume total de poros (VTP); índice de floculação (IF); diâmetro médio geométrico de agregados (DMG); permeabilidade do solo a água (KS); perda de solo (SOLO); perda de água (ÁGUA); índice de qualidade integrado (IQI); índice de qualidade Nemoro (IQN); componente principal 1 (PC1); e componente principal 2 (PC2). 
à manutenção das funções principais do solo, e indicam que o cultivo de eucalipto em escala comercial, quando realizado de forma adequada, pode elevar qualidade do solo e reduzir as perdas por erosão hídrica, principalmente em áreas com histórico de degradação, como a deste estudo.

Em seguida, em ordem decrescente da qualidade do solo está ES, que ficou a frente apenas do SD, que apresentou os piores resultados; reflexo, principalmente, do baixo teor de MOS, que é menor em condições estressantes (Marchiori Júnior e Melo, 1999; Souza e Melo, 2003).

\section{CONCLUSÕES}

Os índices de qualidade do solo, IQI e IQN mostraram-se sensíveis às variações nos diferentes tipos de manejo do solo, quanto a erosão hídrica.

De acordo com o IQI e IQN, a qualidade do solo apresentou gradiente crescente no sentido solo descoberto, plantio do eucalipto em nível sem resíduo, plantio do eucalipto em desnível, plantio do eucalipto em nível com resíduo e vegetação nativa. Essa ordem foi inversa quanto às perdas de solo e água.

O cultivo mínimo do eucalipto contribuiu para que os IQS aproximassem aos do solo com vegetação nativa, evidenciando a importância da manutenção do resíduo sobre o solo.

Os métodos utilizados na indexação dos indicadores de qualidade do solo, IQI e IQN apresentaram elevada correlação. Entretanto, o IQN é o recomendável para as avaliações da qualidade do solo por eliminar o viés do pesquisador, na atribuição dos pesos aos atribuídos do solo.

\section{REFERÊNCIAS}

Alvarenga CC, Mello CR, Mello JM, Silva AM, Curi N. Índice de qualidade do solo associado à recarga de água subterrânea (IQSRA) na bacia hidrográfica do Alto Rio Grande, MG. R Bras Ci Solo. 2012;36:1608-19.

Andrews SS, Carroll R. Designing a soil quality assessment tool for sustainable agro-ecosystem management. Ecol Appl. 2001;11:1573-85.

Andrews SS, Karlen DL, Mitchell JP. A comparison of soil quality indexing methods for vegetable production systems in Northern California. Agric Ecosyst Environ. 2002;90:25-45.

Armenise E, Redmile-Gordon MA, Stellacci AM, Ciccarese A, Rubino P. Developing a soil quality index to compare soil fitness for agricultural use under different managements in the Mediterranean environment. Soil Till Res. 2013;130:91-98.

Bhardwaj AK, Jasrotia P, Hamiltona SK, Robertson GP. Ecological management of intensively cropped agro-ecosystems improves soil quality with sustained productivity. Agric Ecosyst Environ. 2011;140:419-29.

Brejda JJ, Karlen DL, Smith JL, Allan DL. Identification of regional soil quality factors and indications in Northern Mississippi Loess Hills and Palouse Prairie. Soil Sci Soc Am J. 2000a;64:2125-35.

Brejda JJ, Moorman TB, Smith JL, Karlen DL, Allan DL, Dao TH. Distribution and variability of surface soil properties at a regional scale. Soil Sci. Soc. Am. J. 2000b;64:974-82.

Conforti M, Buttafuoco G, Leone AP, Aucelli PPC, Robustelli G, Scarciglia F. Studying the relationship between water-induced soil erosion and soil organic matter using Vis-NIR spectroscopy and geomorphological analysis: A case study in southern Italy. Catena. 2013;110:44-58.

Ditzler CA, Tugel AJ. Soil quality field tools: Experiences of USDA-NRCS Soil Quality Institute. Agron J. 2002;94:33-8.

Doran JW, Jones AJ. Methods for assessing soil quality. Madison: Soil Science Society of America; 1996. (Special publication, 49).

Doran JW, Parkin TB. Defining and assessing soil quality. In: Doran JW, Coleman, DC, Bezdicek DF, Stewart BA, editors. Defining soil quality for sustainable environment. Madison: Soil Science Society of America, 1994. p.3-21.

Freitas DAF, Silva MLN, Cardoso EL, Curi N. Índices de qualidade do solo sob diferentes sistemas de uso e manejo florestal e cerrado nativo adjacente. R Ci Agron. 2012;43:417-28.

Frye WW, Ebelhar SA, Mudorck LW, Blevis RL. Soil erosion effects on properties and productivity of two Kentucky soils. Soil Sci Soc Am J. 1982;46:1051-5.

Karlen DL, Gardner JC, Rosek MJ. A soil quality framework for evaluating the impact of CRP. J Prod Agric. 1998;11:56-60.

Karlen DL, Mausbach MJ, Doran JW, Cline RG, Harris RF, Schumman GE. Soil quality: A concept, definition, and framework for evaluation. Soil Sci Soc Am J. 1997;61:4-10.

Liebig MA, Varvel GE, Doran JW. A simple performance-based index for assessing multiple agroecosystem functions. Agron J. 2001;93:313-8.

Mabit L, Bernard C. Spatial distribution and content of soil organic matter in an agricultural field in eastern Canada, as estimated from geostatistical tools. Earth Surf Proc Land. 2010;35:278-83.

Marchiori Júnior M, Melo WJ. Carbono, carbono da biomassa microbiana e atividade enzimática em um solo sob mata natural, pastagem e cultura do algodoeiro. R Bras Ci Solo. 1999;23:257-63.

Marzaioli R, D’Ascoli R, De Pascale RA, Rutigliano FA. Soil quality in a Mediterranean area of Southern Italy as related to different land use type. Appl Soil Ecol. 2010;44:205-12.

Nuria R, Jérôme M, Léonide C, Christine R, Gérard H, Etienne I, Patrick L. IBQS: A synthetic index of soil quality based on soil macro-invertebrate communities. Soil Biol Biochem. 2011;43:2032-45.

Nizeyimana E, Olson KR. Chemical, mineralogical, and physical property differences between moderately and severely eroded Illinois soils. Soil Sci Soc Am J. 1988;52:1740-48. 
Qi Y, Darilek JL, Huang B, Zhao Y, Sun W, Gu Z. Evaluating soil quality indices in an agricultural region of Jiangsu Province, China. Geoderma. 2009;149:325-34

Qin MZ, Zhao J. Strategies for sustainable use and characteristics of soil quality changes in urban-rural marginal area: A case study of Kaifeng. Acta Geogr Sinica. 2000;55:545-54

Rahmanipour F, Marzaioli R, Bahrami HA, Fereidouni $\mathrm{Z}$, Bandarabadi SR. Assessment of soil quality indices in agricultural lands of Qazvin Province, Iran. Ecol Indic. 2014;40:19-26.

Rhoton FE, Tyler DD. Erosion induced changes in soil properties of a fragipan soil. Soil Sci Soc Am J. 1990;54:223-8.
Souza WJO, Melo WJ. Matéria orgânica em um Latossolo submetido a diferentes sistemas de produção de milho. $\mathrm{R}$ Bras Ci Solo. 2003;27:1113-22.

Sparovek G, Jong van Lier Q, Dourado Neto D. Computer assisted Köppen climate classification: a case study for Brazil. Int J Climatol. 2007;27:257-66.

Sun B, Zhou S, Zhao Q. Evaluation of spatial and temporal changes of soil quality based on geostatistical analysis in the hill region of subtropical China. Geoderma. 2003;115:85-99.

van der Ploeg RR, Böhm W, Kirkham MB. On the origin of the theory of mineral nutrition of plants and the law of the minimum. Soil Sci Soc Am J. 1999;63:1055-62. 